\title{
Perfil do atendimento de urgência e emergência em uma base do noroeste do Paraná
}

\author{
Profile of urgent and emergency care in a northwestern Paraná base \\ Perfil de atención de urgencia y emergencia en una base del noroeste de Paraná
}

Neide Derenzo

ORCID: https://orcid.org/0000-0002-7771-8163 Universidade Estadual do Paraná, Brasil E-mail: neidederenzo@hotmail.com

Daiany Joice Cuba de Miranda ORCID: https://orcid.org/0000-0002-6320-1008 Universidade Estadual do Paraná, Brasil E-mail: daiany_joyce@hotmail.com

Samuel Luciano Pereira da Silva

ORCID: https://orcid.org/0000-0003-2526-446X Universidade Estadual do Paraná, Brasil

E-mail: conutorsamuelsamu192@gmail.com

Igor Fernando Neves

ORCID: https://orcid.org/0000-0001-6914-8220 Universidade Estadual do Paraná, Brasil E-mail: igorneves.fer@gmail.com

Renata Rodrigues Mendonça

ORCID: https://orcid.org/0000-0001-6467-1469 Universidade Estadual do Paraná, Brasil E-mail: re_rodrigues1992@hotmail.com

Drielly Lima Valle Folha Salvador

ORCID: https://orcid.org/0000-0001-9661-598X Universidade Estadual do Paraná, Brasil E-mail: drielly.vale@hotmail.com

Heloá Costa Borim Christinelli

ORCID: https://orcid.org/0000-0003-0772-4194 Universidade Estadual do Paraná, Brasil E-mail: heloa.borim@hotmail.com

Kely Paviani Stevanato

ORCID: https://orcid.org/0000-0003-1872-8246 Universidade Estadual do Paraná, Brasil E-mail: kelystevanato@gmail.com

\begin{abstract}
Resumo
Objetivo: Analisar o perfil epidemiológico das ocorrências de uma base descentralizada do SAMU 192 em um município do Noroeste do Paraná. Metodologia: O presente estudo é retrospectivo, analítico, de abordagem quantitativa. Para tal, foram utilizadas as fichas de atendimentos realizados entre janeiro a março de 2019. Foram coletados dados referentes às características sociodemográficas das vítimas e os tipos de atendimentos de urgência e emergência realizados pelos profissionais. O Presente estudo foi submetido e aprovado pelo Comitê de Ética em Pesquisa da Universidade Estadual do Paraná, respeitando os preceitos éticos da Resolução 466/2012 e 510/2016 do Conselho Nacional de Saúde, sob protocolo de aprovação no 3.313.124 Resultados: Observou que das 941 fichas de atendimentos analisadas $653(69,4 \%)$ por causas clínicas, $196(20,8 \%)$ por traumas, $62(6,6 \%)$ ocorrências psiquiátricas e $30(3,2 \%)$ causas gineco-obstétricas. Evidenciou diferença estatística significativa na comparação entre os sexos, em relação à natureza da ocorrência e o tipo de ambulância disponibilizada para o atendimento. Além disso, os homens atendidos pelo SAMU, apresentaram 1,5 vezes mais chances de demandar de atendimento de uma USA. Conclusões: A análise mostrou a necessidade da elaboração de ações intersetoriais de promoção e prevenção a saúde relacionado a informação sobre os sinais e sintomas característicos de urgência e emergência.
\end{abstract}

Palavras-chave: Atendimento de emergência; Atendimento pré-hospitalar; Causas externas.

\begin{abstract}
Objective: To analyze the epidemiological profile of the occurrences of a decentralized base of SAMU 192 in a municipality in the Northwest of Paraná. Methodology: The present study is retrospective and, analytical, epidemiological study with a quantitative approach. For this purpose, the forms of care provided between January and March 2019 were used. Data were collected regarding the sociodemographic characteristics of the victims and the types of urgent and emergency care provided by the professionals. The present study was submitted to and approved by the
\end{abstract}


Research Ethics Committee of the State University of Paraná, respecting the ethical precepts of Resolution 466/2012 and 510/2016 of the National Health Council, under approval protocol No. 3,313,124 Results: It was observed that of the 941 medical records analyzed $653(69.4 \%)$ due to clinical causes, $196(20.8 \%)$ due to trauma, $62(6.6 \%)$ psychiatric occurrences and 30 (3.2\%) gynecological causes -obstetric. It showed a statistically significant difference in the comparison between the sexes, in relation to the nature of the occurrence and the type of ambulance available for care. In addition, men served by SAMU were 1.5 times more likely to demand assistance from a USA. Conclusions: The analysis showed the need to develop intersectoral health promotion and prevention actions related to information about the characteristic signs and symptoms of urgency and emergency.

Keywords: Emergency care; Pre hospital care; External causes.

\section{Resumen}

Objetivo: Analizar el perfil epidemiológico de la ocurrencia de una base descentralizada del SAMU 192 en un municipio del Noroeste de Paraná. Metodología: El presente estudio es retrospectivo, analítico, analítico con enfoque cuantitativo. Para ello, se utilizaron las formas de atención brindada entre enero y marzo de 2019. Se recolectaron datos sobre las características sociodemográficas de las víctimas y los tipos de atención de urgencia y emergencia brindada por los profesionales. El presente estudio fue sometido y aprobado por el Comité de Ética en Investigación de la Universidad Estadual de Paraná, respetando los preceptos éticos de la Resolución 466/2012 y 510/2016 del Consejo Nacional de Salud, bajo protocolo de aprobación No. 3.313.124. Resultados: Se observó la de las 941 historias clínicas analizadas $653(69,4 \%)$ por causas clínicas, $196(20,8 \%)$ por traumatismo, $62(6,6 \%)$ ocurrencias psiquiátricas y $30(3,2 \%)$ causas ginecológicas obstétricas. Mostró una diferencia estadísticamente significativa en la comparación entre los sexos, en relación con la naturaleza del suceso y el tipo de ambulancia disponible para la atención. Además, los hombres atendidos por SAMU tenían 1,5 veces más probabilidades de exigir ayuda de EE. UU. Conclusiones: El análisis mostró la necesidad de desarrollar acciones intersectoriales de promoción y prevención de la salud relacionadas con la información sobre los signos y síntomas característicos de urgencia y emergencia.

Palabras clave: Atención de emergencia; Atención prehospitalaria; Causas externas.

\section{Introdução}

O crescimento rápido e desorganizado das grandes metrópoles, o desenvolvimento populacional e a globalização, têm ocasionado o aumento da ocorrência de diversas patologias, em especial as de caráter de urgência e emergência, no qual gera alterações epidemiológicas e o desenvolvido de serviços e sistemas que atendam a essa diligência (Almeida,2016).

Frente a esta realidade houve uma crescente transição demográfica no país, acompanhada do aumento das doenças crônicas, nas quais aumentam o risco de situações agudas, em especial as pertinentes ao aparelho circulatório, como o infarto agudo do miocárdio (IAM) e o acidente vascular cerebral (AVC). Somado a isso, o transporte público ineficiente com o crescimento demasiado das frotas de automóveis e motocicletas, juntamente com a violência urbana em grandes centros, são fatores que contribuem para crescentes taxas de morbimortalidade por causas externas que acentuam o desafio no atendimento de urgência e emergência em saúde, segundo o Ministério da Saúde, 2011 e ortiga et al 2016.

Segundo a OMS, estima-se que em 2020, os acidentes de trânsito representarão a segunda causa de morte prematura no mundo. Diante deste alto índice de mortalidade e morbidade por causas externas e pelas doenças crônicas, o Ministério da Saúde (MS) instituiu a Política Nacional de Atenção às Urgências (PNAU), descrita na Portaria nº 1863/GM em 29 de setembro de 2003, devendo ser implantada nas unidades federativas respeitando as competências das três esferas de gestão.

Diante desse cenário o Ministério da Saúde (MS) propõe a implantação das Redes de Atenção à Saúde (RAS), com propósito de fornecer assistência integral e maior articulação entre as complexidades de atendimentos (Almeida,2016). Dentre as RAS, a Rede de Atenção às Urgências (RUE) que tem a função de articular todos os setores da saúde, por meio da ampliação e qualificação do acesso aos usuários, promovendo a humanização e integralidade do cuidado em situação de urgência e emergência nos serviços de saúde, de forma ágil e oportuna (Ministério da Saúde, Portaria n 4.279, de 30 de dezembro de 2010).

A nível nacional, as RAS são compostas pelos dispositivos de saúde que se estendem desde a Atenção Primária à Saúde até ao Atendimento Domiciliar, englobando a Central de Regulação Médica de Urgências, Serviço de Atendimento Móvel de Urgência (SAMU 192), salas de estabilização e UPAs, sendo o SAMU integrante assistencial da RAS que tem como finalidade alcançar o mais rápido possível a pessoa que sofre agravo a saúde encaminhado ambulâncias equipado por profissionais 
capacitados, estando em articulação com todas as redes de saúde Sistema Único de Saúde (Michilin et al, 2016)

Dentro deste contexto, o Atendimento Pré-Hospitalar (APH), SAMU 192, está subdividido em suporte básico e suporte avançado de vida. Define-se como Suporte Básico de Vida (SBV) a estrutura de apoio oferecida às vítimas com risco de agravos desconhecidos e com menor gravidade, executado por profissionais técnicos de enfermagem e um condutor/socorrista, por meio de intervenções menos invasivas. Os eventos de baixa complexidade destacam-se em sua maioria, pois se apresentam em maior número dos atendimentos.

O SBV pode, ainda, atender vítimas em estado grave, como apoio às viaturas de Suporte Avançado de Vida (SAV). As vítimas de maior gravidade obrigatoriamente utilizam-se viaturas de SAV, funcionando como uma Unidade de Terapia Intensiva móvel, (UTI Móvel).

Nos países desenvolvidos houve a implantação do atendimento pré-hospitalar (APH) como um importante serviço de atenção às urgências e emergências. O termo APH refere-se à assistência realizada às vítimas de agravos emergentes e urgentes à condição de saúde, fora do âmbito hospitalar, para a manutenção da vida e diminuição de sequelas (Almeida,2016).

Considera-se que as situações de urgência e emergência são complexas e imprevisíveis, e ainda assim, há escassez de estudos sobre o tema, especialmente do Noroeste do Paraná, já que a implantação dos serviços do SAMU é recente na região estudada. Portanto, o presente estudo tem como objetivo analisar o perfil epidemiológico das ocorrências de uma base descentralizada do SAMU 192 em um município de médio porte do Noroeste do Paraná.

\section{Metodologia}

O presente estudo é retrospectivo e analítico, com abordagem quantitativa, realizado no Serviço de Atendimento Móvel de Urgência (SAMU 192), sendo a população-alvo constituída daquelas vítimas que solicitaram esse serviço, independente do motivo da ocorrência, no período entre janeiro e março de 2019, em um município de médio porte localizado na região do Noroeste do Paraná.

O universo amostral foi identificado por meio das Fichas de Atendimento (FA), mecanismo utilizado para todos os atendimentos de viaturas de Unidades Suporte Avançada (USA) e Unidade de Suporte Básico (USB), pertinentes aos atendimentos prestados e preenchidas pelos integrantes da equipe.

As FA são compostas por informações de identificação do usuário como nome, endereço, sexo e idade, descrição da avaliação primária da vítima (natureza da ocorrência e condições neurológicas, circulatórias e respiratórias), desfecho do atendimento, sinais vitais, histórico de medicação, procedimentos e medicações feitas pela equipe, descrição do acontecimento e dirigentes responsável pelo atendimento.

Neste estudo, foram utilizados dados referentes ao sexo e idade da vítima, categorizadas em crianças (subdividida em 3 categorias sendo <1 ano, 2-4 anos, e 5-9 anos), adolescentes (10-19 anos), adultos (20 a 59 anos) e idosos (> 60 anos), à natureza da ocorrência: clínica, psiquiátrica, gineco-obstétrica e/ou trauma, qual ambulância foi enviada à ocorrência: USA e/ou USB, o desfecho do atendimento e a localização do chamado conforme o bairro. O colhimento do material foi feito pelos pesquisadores principais deste estudo.

Os critérios de inclusão foram: todas as FA que estavam com os campos completos, assinados pela equipe e com letras legíveis. Excluíram-se as FA que foram acionadas por indivíduos que pertenciam a outros municípios. Também se excluiu as FA com ilegibilidade e sem assinaturas da equipe responsável.

A tabulação dos dados seu deu por meio da planilha do Microsoft Excel® 2016 e exportados para o software IBM SPSS ${ }^{2} 20.0$ para análise descritiva e inferencial. Para a comparação entre os grupos independentes foi utilizado o Teste T de Student, e a regressão logística uni variada, por meio do teste Qui-quadrado, com cálculo de Razão de Chances (RC) e Intervalo 
de Confiança (IC) (95\%) para verificar associação entre as variáveis. Considerou-se o valor de $p \leq 0,05$ para significância estatística.

A presente pesquisa foi submetida e aprovada pelo Comitê de Ética em Pesquisa da Universidade Estadual do Paraná, respeitando os preceitos éticos da Resolução 466/2012 e 510/2016 do Conselho Nacional de Saúde, sob protocolo de aprovação $\mathrm{n}^{\circ}$ 3.313.124.

\section{Resultados}

Entre janeiro e março de 2019 ocorreram 1.247 atendimentos, destes, 941 foram analisados neste estudo. A maioria das ocorrências foram de atendimentos a vítimas do sexo feminino, com 50,8\% (478). Em relação aos atendimentos por idade houve predomínio de vítimas em idade produtiva entre 20 e 59 anos (Tabela 1).

Tabela 1 - Atendimentos realizados pelo SAMU 192, de acordo com faixa etária e sexo das ocorrências entre janeiro e março de 2019. Noroeste PR, 2019.

\begin{tabular}{|c|c|c|c|c|c|c|c|}
\hline \multirow{3}{*}{ Variável } & & \multicolumn{4}{|c|}{ Sexo } & \multirow{2}{*}{\multicolumn{2}{|c|}{ Total }} \\
\hline & & \multicolumn{2}{|c|}{$\mathrm{M}$} & \multicolumn{2}{|c|}{$\mathrm{F}$} & & \\
\hline & & $\mathbf{N}$ & $\%$ & $\mathbf{n}$ & $\%$ & $\mathbf{n}$ & $\%$ \\
\hline \multirow{6}{*}{ Faixa etária } & $0-1$ ano & 12 & 2,6 & 3 & 0,6 & 15 & 1,59 \\
\hline & $2-4$ anos & 4 & 0,9 & 6 & 1,3 & 10 & 1,06 \\
\hline & $5-9$ anos & 6 & 1,3 & 6 & 1,3 & 12 & 1,28 \\
\hline & $10-19$ anos & 29 & 6,3 & 49 & 10,3 & 78 & 8,29 \\
\hline & $20-59$ anos & 238 & 51,4 & 222 & 46,4 & 460 & 48,88 \\
\hline & $\geq 60$ anos & 174 & 37,6 & 192 & 40,2 & 366 & 38,89 \\
\hline Total por sexo & & 463 & 49,2 & 478 & 50,8 & 941 & 100 \\
\hline
\end{tabular}

Legenda: n: número absoluto \%: percentual, M: masculino e F: feminino.

Fonte: Base SAMU - 192(2019).

Em relação à natureza da ocorrência, a maioria dos atendimentos foi de natureza clínica, correspondendo a 69,6\% (655) do total. Entretanto, evidenciou-se que algumas variáveis tiveram comportamentos diferentes quando analisada pelo sexo das vítimas. Entre o sexo feminino, atendimentos de natureza psiquiátrica, com 70,9\% (44) dos atendimentos (Tabela 2). Já entre o sexo masculino, os atendimentos decorrentes de traumas 62,2\% (122). Já a variável atendimento de natureza clínica foi a que obteve maior número de ocorrências para ambos os sexos.

Ainda sobre a Tabela 2, comparou-se a natureza da ocorrência para a variável idade no qual observou-se que a natureza de ocorrência clínica concentrou a maior taxa de ocorrências do total de atendimentos prestados com predominância para idade $\geq 60$ anos, 46,7\% (306), já para a variável de natureza trauma, o predomínio foi para a faixa etária de 20-59 anos 60,2\% (118), nesta mesma faixa etária observou-se também a predominância da natureza psiquiátrica 63\% (39). 
Tabela 2 - Atendimentos realizados pelo SAMU 192, natureza da ocorrência, de acordo com sexo e faixa etária das ocorrências entre janeiro e março de 2019. Noroeste-PR, 2019.

\begin{tabular}{|c|c|c|c|c|c|c|c|c|c|c|c|}
\hline \multirow{3}{*}{ Variável } & & \multicolumn{8}{|c|}{ Natureza da Ocorrência } & & \\
\hline & & \multicolumn{2}{|c|}{ Clínica } & \multicolumn{2}{|c|}{ Psiquiátrica } & \multicolumn{2}{|c|}{ Trauma } & \multicolumn{2}{|c|}{ GO } & \multicolumn{2}{|c|}{ Total } \\
\hline & & $\mathbf{n}$ & $\%$ & $\mathbf{n}$ & $\%$ & $\mathbf{N}$ & $\%$ & $\mathbf{N}$ & $\%$ & $\mathbf{n}$ & $\%$ \\
\hline \multirow{2}{*}{ Sexo } & Feminino & 332 & 50,7 & 44 & 70,9 & 74 & 37,8 & 28 & 100 & 478 & 50,95 \\
\hline & Masculino & 323 & 49,3 & 18 & 29,1 & 122 & 62,2 & - & - & 463 & 49,4 \\
\hline \multirow{6}{*}{$\begin{array}{l}\text { Faixa } \\
\text { etária }\end{array}$} & $0-1$ ano & 13 & 2,0 & 0 & 0,0 & 2 & 1,0 & 0 & 0,0 & 15 & 1,6 \\
\hline & $2-4$ anos & 7 & 1,1 & 1 & 1,6 & 2 & 1,0 & 0 & 0,0 & 10 & 1,1 \\
\hline & 5-9 anos & 6 & 0,9 & 0 & 0,0 & 6 & 3,1 & 0 & 0,0 & 12 & 1,3 \\
\hline & 10-19 anos & 31 & 4,7 & 10 & 16,1 & 20 & 10,2 & 17 & 60,7 & 78 & 8,3 \\
\hline & 20-59 anos & 292 & 44,6 & 39 & 63,0 & 118 & 60,2 & 11 & 39,3 & 460 & 48,9 \\
\hline & $\geq 60$ anos & 306 & 46,7 & 12 & 19,3 & 48 & 24,5 & 0 & 0,0 & 366 & 38,8 \\
\hline \multicolumn{12}{|l|}{ Total por } \\
\hline $\begin{array}{l}\text { faixa } \\
\text { etária }\end{array}$ & & 655 & 100 & 62 & 100 & 196 & 100 & 28 & 93 & 941 & 100 \\
\hline
\end{tabular}

Legenda: n: número absoluto \%: percentual, M: masculino e F: feminino.

Fonte: Os autores.

À análise, o município do Noroeste do Paraná é composto por 46 bairros e 6 distritos (Figura 1), sendo que sua distribuição espacial por meio do mapa de Kernel ou mapa de calor, permite perceber que a região central, sudoeste e sudeste foram as principais responsáveis por solicitar os atendimentos do serviço.

Figura 1 - Mapa de Kernel da distribuição espacial dos chamados pelo serviço de urgência e emergência do SAMU 192 no município de Paranavaí/PR, 2019. (n=941).

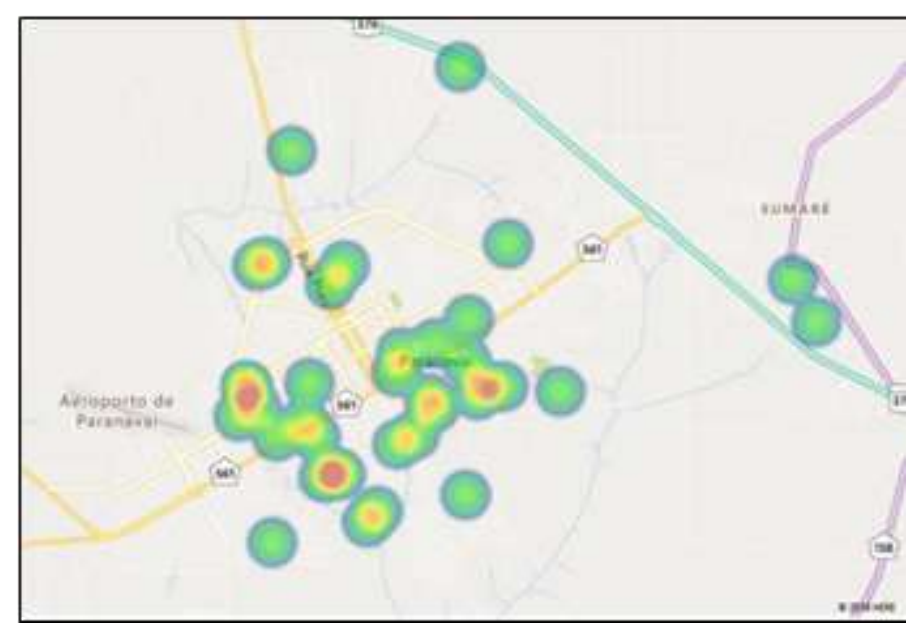

Fonte: Os autores (2019). 
Cabe citar que um distrito do município não aparece no mapa para melhor visualização da região central e metropolitana. No mapa, as cores mais quentes revelam maior taxa de chamados pelo serviço, independente da natureza da ocorrência (Figura $1)$.

Dentre os atendimentos, a maior parte não teve a causa base do chamado especificada, identificadas apenas como “outros", configurando um viés de causa em $21,2 \%$ (200) fichas e sequencialmente, a causa mais prevalente no estudo foram as de natureza clínica.

Entre os atendimentos por traumas, a causa mais recorrente foi por Quedas inespecíficas com 10,2\% (96) atendimentos, seguida de crises convulsivas 7,7\% (72) e síncope 6,4\% (60) ocorrências. Dos atendimentos gineco-obstétricos, a prevalência foi por complicações da gestação, e os de natureza psiquiátrica, prevaleceram os chamados por alterações por uso de drogas ilícitas.

Quanto ao tipo de viatura de suporte utilizado para atender as ocorrências realizadas no período do estudo, destaca-se a apresentação das Unidades de Suporte Básico (USB), com 87,9\% (827) das ocorrências atendidas. Logo, as Unidades de Suporte Avançado (USA), utilizadas para a assistência de vítimas com maior gravidade, consistiu em 12,1\% (114) das ocorrências e se relacionaram positivamente com os chamados de natureza psiquiátrica. Outro dado apresentado é a relação entre o alto número de transferências realizadas pela USA e um menor número de atendimentos realizados pela mesma.

De acordo com as informações apresentadas na Tabela 3, no que se refere ao desfecho do atendimento, 51,1\% (481) das ocorrências atendidas foram encaminhadas para Unidade de Pronto Atendimento (UPA) e 23,1\% (217) dos atendidos tiveram como conclusão da atenção pré-hospitalar encaminhados para o Hospital Santa Casa.

Tabela 3 - Atendimento realizados pelo SAMU 192, sexo x principais queixas, natureza da ocorrência, tipo de ambulância, desfecho de atendimento.

\begin{tabular}{|c|c|c|c|}
\hline Variáveis & $\mathbf{N}(\%)$ & $\mathbf{N}(\%)$ & p-valor \\
\hline Principais Queixas $(n=941)$ & $\mathrm{M}$ & $\mathrm{F}$ & \\
\hline Outros & $111(24,0)$ & $89(18,6)$ & \\
\hline Queda Inespecífica & $57(12,3)$ & $39(8,2)$ & \\
\hline Crise Convulsiva & $39(8,4)$ & $33(6,9)$ & \\
\hline Sincope & $26(5,6)$ & $34(7,1)$ & \\
\hline Mal-estar & $21(4,5)$ & $38(7,9)$ & \\
\hline Parada Cardiorrespiratória & $20(4,3)$ & $9(1,9)$ & \\
\hline Acidente - Automobilístico & $19(4,1)$ & $9(1,9)$ & \\
\hline Embriaguez & $19(4,1)$ & $13(2,7)$ & \\
\hline Dispneia & $18(3,9)$ & $27(5,6)$ & \\
\hline Dor Abdominal & $17(3,7)$ & $12(2,5)$ & \\
\hline Dor Precordial & $16(3,5)$ & $19(4,0)$ & \\
\hline Suspeita de Acidente Vascular Encefálico & $14(3,0)$ & $8(1,7)$ & \\
\hline Ferimento Corto-Contuso & $11(2,4)$ & $2(0,4)$ & \\
\hline Acidente - Motocicleta & $10(2,2)$ & $10(2,1)$ & \\
\hline Surto Psicótico & $9(1,9)$ & $12(2,5)$ & \\
\hline Agressão física & $8(1,7)$ & $4(0,8)$ & \\
\hline Hipertensão Arterial & $8(1,7)$ & $11(2,3)$ & \\
\hline
\end{tabular}




\begin{tabular}{|c|c|c|c|}
\hline Intoxicação Exógena & $8(1,7)$ & $11(2,3)$ & \\
\hline Cefaleia & $6(1,3)$ & $3(0,6)$ & \\
\hline Hiperglicemia & $6(1,3)$ & $7(1,5)$ & \\
\hline Tentativa de Suicídio & $6(1,3)$ & $13(2,7)$ & \\
\hline Hipoglicemia & $5(1,1)$ & $15(3,1)$ & \\
\hline Hipotensão & $5(1,1)$ & $7(1,5)$ & \\
\hline Crise Nervosa/Ansiedade & $2(0,4)$ & $19(4,0)$ & \\
\hline Dor na Coluna & $2(0,4)$ & $6(1,3)$ & \\
\hline Gestante & & $28(5,9)$ & \\
\hline \multicolumn{4}{|l|}{ Natureza da ocorrência } \\
\hline Clínica & $341(45,8)$ & $404(54,2)$ & \\
\hline Trauma & $122(62,2)$ & $74(37,8)$ & $<0,001^{*}$ \\
\hline \multicolumn{4}{|l|}{ Tipo de ambulância disponibilizada } \\
\hline Unidade de Suporte Básico & $396(85,5)$ & $431(90,2)$ & \\
\hline Unidade de Suporte Avançado & $67(14,5)$ & $47(9,8)$ & $0,029^{*}$ \\
\hline \multicolumn{4}{|l|}{ Desfecho do atendimento } \\
\hline Encaminhamento ao UPA & $234(50,5)$ & $247(51,7)$ & \\
\hline Encaminhamento a Santa Casa & $94(20,3)$ & $123(25,7)$ & \\
\hline Liberado no Local & $46(9,9)$ & $47(9,8)$ & \\
\hline Recusa do Atendimento & $32(6,9)$ & $29(6,1)$ & \\
\hline Transferência PAM/Santa Casa & $35(7,6)$ & $22(4,6)$ & \\
\hline Óbito & $16(3,5)$ & $4(0,8)$ & \\
\hline Solicitado apoio da UPA & $1(0,2)$ & $2(0,4)$ & \\
\hline Transferência Santa Casa/Aeroporto & $1(0,2)$ & $2(0,4)$ & \\
\hline Ocorrência cancelada & $0(0,0)$ & $1(0,2)$ & \\
\hline Socorrido por terceiros & $1(0,2)$ & $1(0,2)$ & \\
\hline Solicitado atendimento aéreo & $1(0,2)$ & $0(0,0)$ & \\
\hline \multicolumn{4}{|l|}{ Cancelamento da ocorrência pelo médico } \\
\hline regulador & $2(0,4)$ & $0(0,0)$ & \\
\hline Total geral & $163(49,2 \%)$ & $478(50,8 \%)$ & \\
\hline
\end{tabular}

Legenda: UPA: Unidade de Pronto Atendimento, USA: Unidade de Suporte Avançado, USB: Unidade de Suporte Básico.Associação significativa (Teste t de Student para comparação entre os sexos e Qui-quadrado para verificação de associação $(\mathrm{p}<0,05)$. Fonte: Autores.

Na comparação entre os sexos, evidenciou-se diferença estatística significativa em relação à natureza da ocorrência e o tipo de ambulância disponibilizada para o atendimento. Esta proporção é de que a frequência de atendimentos de urgência por trauma seja de dois homens para cada mulher, independente da faixa etária. Além disso, os homens atendidos pelo SAMU, apresentaram 1,5 vezes mais chances de demandar atendimento de uma USA.

Imprescindível citar que as fichas preenchidas de forma incompleta e ilegível representaram 24,5\%. Destacando-se um alto índice, uma vez que esse instrumento é utilizado como identificação básica do paciente e seu quadro clínico. 


\section{Discussão}

As situações de urgência e emergência são complexas e imprevisíveis, e caracterizam-se como um tema escasso no campo de estudo no Noroeste do Paraná, já que a implantação dos serviços do SAMU é recente na região estudada. Com isso analisar o perfil epidemiológico das ocorrências em uma base descentralizada do SAMU 192 é de suma importância na contribuição de dados que possam auxiliar na formulação de ações de promoção e prevenção de agravos em saúde.

Pode-se observar neste estudo uma maior taxa de ocorrências em população economicamente ativa (20-59 anos), que necessita frequentemente do serviço de emergência pré-hospitalar, corroborando com uma pesquisa de Jequié, Bahia, em 2014 que reforça os atendimentos destinados a este grupo (Lefunded et al,2016). Além disso, a ocorrência de maior número de casos de trauma e acidentes de trânsito foi evidenciada no sexo masculino, fato que coincide com um estudo realizado nos Estados Unidos da América (EUA), em que o sexo masculino representou 56,0\% dos atendimentos pelo serviço móvel (Riney,2019) e segundo a Organização Mundial da Saúde, homens jovens tem cerca de três vezes mais chances de morrer por acidentes de trânsito e maior prevalência de ocorrências desta natureza também foi observada em outros estudos no país (Franco et al,2015) (Castro, Faustino, Ribeiro, 2020) . Nas mulheres, a natureza gineco-obstétrica todas estavam gestantes e a idade destas variou entre 10 a 59 anos. Dentre a faixa etária encontrada, evidencia-se um dos agravos de saúde pública, a gestação na adolescência, fator condicionante que aumenta o risco de intercorrências pré-natais.

A segunda ocorrência de maior número de casos foram quedas inespecíficas, coincide com estudos já realizados onde esse é um trauma muito frequente em idosos possuindo sua prevalência em ambiente domiciliar. A maior complicação das vítimas idosas de queda são as lesões físicas, sendo a mortalidade com maior incidência a traumas de quadril e crânio encefálico atingindo índice de 9\% de mortalidade no mundo (Riney et al,2019), (Ribeiro et al,2016), (Tiensoli et al, 2019).

Dentre as causas analisadas, a maioria dos atendimentos foi de natureza clínica, como as crises convulsivas, corroborando com outros estudos (Tibães et al, 2018), (Silva et al, 2020). Os atendimentos de natureza psiquiátrica, prevaleceram os chamados por alterações por uso de drogas ilícitas, corroborando com os outros achados (Almeida,2016).

Pesquisas realizadas nos estados brasileiros apontam os agravos clínicos como maior número de casos, sendo a crise convulsiva relatada em São Paulo com maior força nos atendimentos realizados pelo SAMU (Almeida,2016). No Paraná estudos realizados mostraram também que grande parte dos atendimentos foram clínicos, dados que corroboram com indicadores nacionais de mortalidade, morbidade, evidenciando que os agravos clínicos são dominantes ( Seyboth et al, 2016)

Ao analisar a viatura de suporte utilizado nas ocorrências as Unidades de Suporte Básico (USB) tiveram o maior número de atendimentos, demonstrando assim que o usuário procura o SAMU em situações que não são emergências, tendo apenas este serviço como referência como única porta de acesso ao serviço de saúde, desconsiderando a atenção primária, que deveria absorver em grande parte esta demanda de baixa complexidade. Os atendimentos da unidade de suporte avançado tem relação às solicitações de natureza psiquiátrica, que podem ser explicadas pela necessidade de contenção física e/ou química que costumam ser frequentes nos atendimentos a pacientes em surtos psiquiátricos e usuários de drogas (Almeida,2016).

Pelo município onde se efetuou o estudo ser um polo, e abarcar o único hospital da cidade de referência para a região, sugere-se que a relação entre o alto número de transferências realizadas pela USA e um menor número de assistência executada, evidencia-se, um problema de cobertura de suporte avançado de vida já que a base possui apenas uma UTI móvel. Este dado reflete novamente, a falta de esclarecimento da população quanto ao real objetivo do SAMU. Estudos realizados em Goiás e também no sul do Brasil apresenta uma discrepância significativa entre as assistências efetuadas pela USB em relação a USA. (Castro, Faustino, Ribeiro, 2020), (Martins et al, 2020)

Em relação ao desfecho do atendimento o estudo mostrou que maior parte das ocorrências foram encaminhadas a Unidade de Pronto Atendimento (UPA), sendo este o local de referência para os atendimentos de urgência e emergência. Uma 
pesquisa feita no estado do Rio Grande do Sul, revela as unidades de pronto atendimentos como principal serviço que recebem os usuários do SAMU, podendo estar relacionado com a referência que são de infraestrutura (Amaral et al, 2016).

$\mathrm{Na}$ comparação entre os sexos, evidenciou-se a relação entre à natureza da ocorrência e o tipo de ambulância disponibilizada para o atendimento, no qual, o sexo masculino utilizou um número maior de atendimentos utilizando o transporte por USA. Esses achados são ratificados em outros estudos, ainda que os homens possuam maiores taxas de mortalidade frente aos agravos, a procura pela assistência primária é de forma tardia, fatos que implicaram decisivamente na criação da Política Nacional de Atenção Integral à Saúde do Homem pelo Ministério da Saúde (Ministério da Saúde,2015) Tais fatores corroboram com a hipótese de que homens procuram o serviço de saúde com manifestações de agravos já instalados. (Battistiet et al, 2019).

Um problema encontrado neste estudo foi o preenchimento incompleto e ilegível de algumas fichas de atendimentos. Um possível fato de não serem preenchidas de forma adequada é a falta de tempo hábil. Podendo correlacionar a este fator a sobrecarga da equipe, levando em consideração que a equipe muitas vezes atua em um ambiente estressante e prestam assistência a pacientes com alto potencial de rebaixamento frente aos agravos (Guibu et al, 2017).

Evidenciou-se que as causas agudas que demandam de atendimento de urgência e emergência acometem, em sua maioria, a população ativa entre 20 e 59 anos, em decorrência de natureza clínica, sendo em sua maioria como principal queixa indícios que caracterizam uma assistência na atenção básica, como por exemplo, cefaleia e mal-estar. A maioria dos indivíduos atendidos foram encaminhados ao pronto atendimento municipal como desfecho da ocorrência.

\section{Conclusão}

Acredita-se que este estudo possa contribuir para a ampliação do conhecimento sobre o uso da rede urgência e emergência SAMU 192, já que grande maioria das queixas foram sensíveis a atenção primaria, além de propor elaboração de ações intersetoriais de promoção e prevenção a saúde relacionadas a informar a população quanto ao tipo de serviço dentre a RAS deve ser acionado para que a qualidade na assistência oferecida a comunidade seja de qualidade.

\section{Referências}

Almeida, P. M. V., Acuqua, M. C. Q. D., Cyrino, C. M. S., et al. (2016). Análise dos atendimentos do SAMU 192: Componente móvel da rede de atenção às urgências e emergências. Esc Anna Nery 2016;20(2):289-295. http://www.scielo.br/scielo.php?pid=S141481452016000200289\&script=sci_abstra ct\&tlng=pt.

Amaral, C. S., Reck Santos, D. L., et al. (2018). Situações de urgência e emergência na atenção primária reguladas pelo SAMU. Journal Health NPEPS. 3(1), 241-52.

Battisti, G. R., Branco, A., Caregnato, R. C. A., \& Oliveira, M. M. C. (2019). Perfil de atendimento e satisfação dos usuários do Serviço de Atendimento Móvel de Urgência (SAMU). Rev. Gaúcha Enferm. 40: e20180431. http://dx.doi.org/10.1590/1983-1447.2019.20180431

Castro, R. R., Faustino, S. U., \& Ribeiro, D. M. (2020). Caracterização das ocorrências do serviço de Atendimento Móvel de Urgência - SAMU. REAEnf/EJNC. Vol. 7. e5625. https://doi.org/10.25248/reaenf.e5625.2020.

Franco, M. S., Lins, A. C., Lima, A. K., \& Araújo, T. L. (2015). Amaral, R.C. Caracterização de pacientes vítimas de acidentes de trânsito admitidos em hospital regional da Paraíba. R Interd ;8(2):123-29. https://revistainterdisciplinar.uninovafapi.edu.br/index.php/revinter/article/view/567 .

Guibu, I. A., Moraes, J. C., Guerra, J. A. A., Costa, E. A., Acurcio, F. A., Costa, K. S., et al. (2017). Main characteristics of patients of primary health care services in Brazil. Rev. Saúde Pública [Internet]. ;51(2):17. http://dx.doi.org/10.11606/s1518-8787.2017051007070.

Lefundes, G. A. A., Gonçalves, N. O., Nery, A. A., Vilela, A. B. A., \& Filho, I. E. M. (2016). Caracterização das ocorrências do serviço de atendimento móvel de urgência. Rev Baiana de Enfermagem; 30(3): 1-10. 10.18471/rbe.v30i3.16387.

Malta, D. C., Andrade, S. S., Gomes, N., Silva, M. M., Morais, O. L., Reis, A. A., et al. (2016). Injuries from traffic accidents and use of protection equipment in the Brazilian population, according to a population-based study. Ciênc saúde coletiva. 2016;21(2):399-410.

Martins, C. F., et al. (2020). Serviço de Atendimento Móvel de Urgência: caracterização dos agravos clínicos. Research, Society and Development, 9(10), e1649107545. http://dx.doi.org/10.33448/rsd-v9i10.7545.

Michilin, N. S., et al. (2016). Análise dos atendimentos obstétricos realizados pelo Serviço de Atendimento Móvel de Urgência. Rev. Bras. Enferm., Brasília;69(4):669-75.http://www.scielo.br/scielo. phpscript=sci_arttext\&pid=S003471672016000400669\&lng=pt\&nrm=iso. 
Research, Society and Development, v. 10, n. 5, e14010514859, 2021

(CC BY 4.0) | ISSN 2525-3409 | DOI: http://dx.doi.org/10.33448/rsd-v10i5.14859

Ministério da Saúde. (2010). Portaria n ${ }^{\circ}$ 4.279, de 30 de dezembro de 2010. Estabelece diretrizes para a organização da Rede de Atenção à Saúde no âmbito do Sistema Único de Saúde [portaria na internet]. Diário Oficial da União 30 dez 2010. http://www.saude.caop.mp.pr.gov. br/arquivos/File/kit_atencao_perinatal/legis/portaria_4279_2010_diretrizes_rede_as.pdf.

Ministério da Saúde. (2011). Portaria no 1.600, de 07 de julho de 2011 - Reformula a Política Nacional de Atenção às Urgências e institui a Rede de Atenção às Urgências no Sistema Único de Saúde (SUS). http://bvsms.saude.gov.br/bvs/saudelegis/gm/2011/prt1600_07_07_2011.html.

Ministério da Saúde. (2015). Secretaria de Atenção à Saúde, Departamento de Ações Programáticas Estratégicas. Política Nacional de Atenção Integral à Saúde do Homem. Brasília (DF). http://www.unfpa.org.br/Arquivos/saude_do_homem.pdf

Ortiga, A. M. B., et al.(2016). Avaliação do Serviço de Atendimento Móvel de Urgência em Santa Catarina. Brasil. Bibl. Virt. Saúde. Cad. Saúde Pública. 2016;32(12). http://pesquisa.bvsalud.org/enfermagem/resource/pt/biblio-828402.

Ribeiro, A. P., Edinilsa, R. S., Carlos, M. A. S., et al. (2016). Quedas acidentais nos atendimentos de urgência e emergência: resultados do VIVA Inquérito de 2014. Ciênc. saúde coletiva [Internet] 2016; 2 (12): 3719-3727. https://doi.org/10.1590/1413-812320152112.18452016.

Riney, L. C., Brokamp, C., Beck, A. F., Pomerantz, W. J., Schwartz, H. P., \& Florin, T. A. (2019). Emergency Medical Services Utilization Is Associated With Community Deprivation in Children. Prehosp Emerg Care, 2019;23(2):225-232. https://doi.org/10.1080/10903127. 2018.1501124.

Seyboth, M. P., Assada, V. K., \& Danielli, V. R. (2016). Delineamento do perfil epidemiológico dos atendimentos do sistema de atendimento móvel de urgência (SAMU) Maringá-PR. Rev. Uningá [Internet] 2016; 48, 51-55. http://revista.uninga.br/index.php/uninga/article/view/1285

Silva, R. S., Davyd, M. S., Leislane, B. S., et al. (2020). O perfil do paciente atendido pelo serviço móvel de urgência e emergência (samu-192) de um município da região norte do país. Rev. Cereus- Especial de Aniversário [Internet]. 12(2) (2020). 10.18605/2175-7275/cereus.v12n2p241-252.

Tibães, H. B. B., Silva, D. M., Alves, M., et al. (2018). Perfil de Atendimento do Serviço de Atendimento Móvel de Urgência no Norte de Minas Gerais. Rev Fund Care Online. 2018 jul./set.; 10(3):675-682. http://dx.doi.org/10.9789/ 2175-5361.2018.v10i3.675-682.

Tiensoli, S. D., Santos, M. L., Moreira, A. D., et al. (2019). Características dos idosos atendidos em um pronto-socorro em decorrência de queda. Rev. Gaúcha Enferm. [Internet]. 40: e20180285. Available from: http://www.scielo.br/scielo.php?script=sci_arttext\&pid=S1983-14472019000100426 \&lng=en. Epub Aug 05, 2019. https://doi.org/10.1590/1983-1447.2019.20180285. 